\title{
Oblique Stimulated Raman Scattering of a Short Laser Pulse in a Plasma Channel
}

\author{
E.J. Turano, C.J. McKinstrie
}

February 22, 2000

U.S. Department of Energy

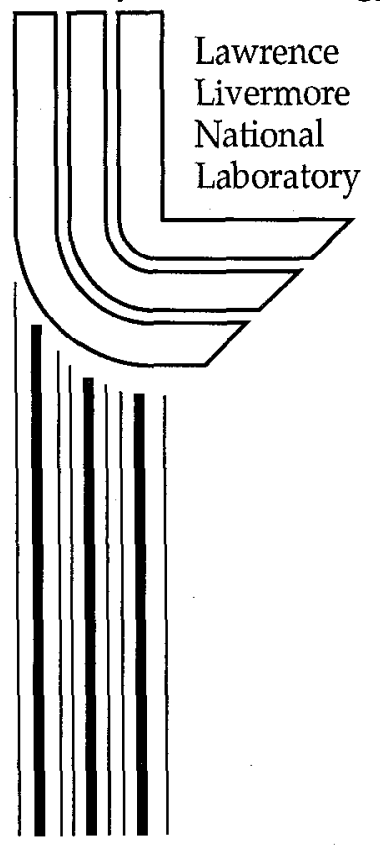

Approved for public release; further dissemination unlimited 


\section{DISCLAIMER}

This document was prepared as an account of work sponsored by an agency of the United States Government. Neither the United States Government nor the University of California nor any of their employees, makes any warranty, express or implied, or assumes any legal liability or responsibility for the accuracy, completeness, or usefulness of any information, apparatus, product, or process disclosed, or represents that its use would not infringe privately owned rights. Reference herein to any specific commercial product, process, or service by trade name, trademark, manufacturer, or otherwise, does not necessarily constitute or imply its endorsement, recommendation, or favoring by the United States Government or the University of California. The views and opinions of authors expressed herein do not necessarily state or reflect those of the United States Government or the University of California, and shall not be used for advertising or product endorsement purposes.

Work performed under the auspices of the U. S. Department of Energy by the University of California Lawrence Livermore National Laboratory under Contract W-7405-Eng-48.

This report has been reproduced

directly from the best available copy.

Available to DOE and DOE contractors from the

Office of Scientific and Technical Information

P.O. Box 62, Oak Ridge, TN 37831

Prices available from (423) 576-8401

http:/ / apollo.osti.gov/bridge/

Available to the public from the

National Technical Information Service

U.S. Department of Commerce

5285 Port Royal Rd.,

Springfield, VA 22161

http://www.ntis.gov/

OR

Lawrence Livermore National Laboratory

Technical Information Department's Digital Library

http://www.llnl.gov/tid/Library.html 
February 22, 2000

\section{OBLIQUE STIMULATED RAMAN SCATTERING OF A SHORT LASER PULSE IN A PLASMA CHANNEL*}

\section{E. J. Turano}

Department of Physics and Astronomy, University of Rochester, Rochester, New York 14627 Laboratory for Laser Energetics, 250 East River Road, Rochester, New York 14623 Current Address:

Lawrence Livermore National Laboratory, 7000 East Avenue, Livermore, California 94550

\section{J. McKinstrie}

Department of Mcchanical Engincering, University of Rochestcr, Rochester, New York 14627 Laboratory for Laser Energetics, 250 East River Road, Rochester, New York 11623

The spatiotemporal evolution of parametric instabilities such as stimulated Raman scattering is studied analytically in time and two spatial dimensions. Initial and boundary conditions are chosen to represent the entrance, propagation, and exit of a laser pulse of finite extent as it progresses through a homogeneous collisional plasma channel. For most scattering angles daughter wave growth is enhanced by lateral reflections within the channel. At late times the two-dimensional interaction approaches a one-dimensional damped mode in which the dissipative loss from lateral transmission of the Stokes wave through the channel boundaries is equivalent to an overall damping of the Stokes amplitudes within the channel.

PACS numbers: $52.35 . \mathrm{Mw}, 52.35 . \mathrm{Nx}, 52.40 . \mathrm{Nk}, 42.65 . \mathrm{Dr}$

* Submitted to Physics of Plasmas 


\section{INTRODUCTION}

Early work on the spatiotemporal evolution of parametric instabilities was described by Bers [1]. Recently, there has been a resurgence of interest in the spatiotemporal evolution of stimulated Raman scattering (SRS) [2-12] and stimulated Brillouin scattering (SBS) [1321], driven by the realization that the transient phase of these instabilities dominates many experiments. This current work serves to expand and unify our previous works $[7,8,10-12]$ by developing an analytic model of parametric instabilities within a plasma channel which includes the effects of two spatial dimensions, damping, finite pulse and plasma boundaries, and oblique reflections of the daughter waves induced by lateral density variations.

Due to the multitude of parametric instabilities, it is impossible to include all of them in this work. Thus, with regard to current interest, we have chosen as an example to consider only that of Stokes generation as applicable to stimulated Raman scattering. The analyses of other parametric instabilities can follow quite easily in a similar fashion.

Stimulated Raman Scattering (SRS) is the decay of an incident light wave (0) into a frequency-downshifted, or Stokes, light wave (1) and Langmuir wave (2) (Fig. 1). The conservation of energy and momentum is reflected in the frequency and wave-vector matching conditions

$$
\omega_{0}=\omega_{1}+\omega_{2}, \quad \mathbf{k}_{0}=\mathbf{k}_{1}+\mathbf{k}_{2}
$$

In the weak-coupling regime, the initial evolution of SRS in a homogeneous two-dimensional collisionless plasma is governed by the linearized equations

$$
\left(\partial_{t} \pm v_{1 y} \partial_{y}+v_{1 x} \partial_{x}\right) A_{+}=\gamma_{0} N_{+}, \quad \partial_{t} N_{+}=\gamma_{0} A_{+}
$$

where $x$ and $y$ are the two spatial dimensions, $t=c t$, positive signs refer to upgoing waves [Fig. 1(a)], negative signs refer to downgoing waves [Fig. 1(b)], $A_{0, \pm}=e A_{0, \pm} / m_{e} c^{2}$ represents the quiver velocity of the Stokes waves, $N_{ \pm}=\left(\omega_{2} / \omega_{1}\right)^{1 / 2} \omega_{2} n / c\left|\mathbf{k}_{2}\right| n_{0}$ represents the plasma density fluctuations of the Langmuir waves, $\gamma_{0}=\omega_{2}\left|\mathbf{k}_{2}\right| A_{0} / 4\left[\omega_{2}\left(\omega_{0}-\omega_{2}\right)\right]^{1 / 2}$ is the temporal growth rate of SRS in an infinite plasma, and the group velocities of- 
the daughter waves are given by $v_{1}=c^{2}\left|\mathbf{k}_{1}\right| / \omega_{1}$ and $v_{2}=3 v_{t}^{2}\left|\mathbf{k}_{2}\right| / 2 \omega_{2}$ where $v_{t}$ is the thermal velocity of the electrons [22]. In consideration of the large velocity of the Stokes wave within the plasma $\left(v_{1 x} \approx c \cos \theta, v_{1 y} \approx c \sin \theta\right.$ for scattering angle $\left.0 \leq \theta \leq \pi\right)$ and the comparably small velocity of the Langmuir wave, we have neglected terms such as $v_{2 x} \partial_{x}$ and $v_{2 y} \partial_{y}$ in the second of Eqs. (2) so that the Langmuir wave does not convect relative to the plasma. Although the governing equations neglect additive stochastic source terms, the closed form analytic solutions for the daughter wave amplitudes that these equations produce have the same gain factor and qualitative spatiotemporal evolution as models which include the stochastic terms [13-15]. Finally, a system such as Eqs. (2) can also be generalized to include both the stabilizing effect of the anti-Stokes (frequency-upshifted) wave $[2,5,9]$ and the effects of strong coupling $[9,16,18,19]$.

\section{TWO-DIMENSIONAL FORWARD SRS}

The governing equations and boundary and initial conditions for two-dimensional forward SRS in a collisionless plasma channel are given by Eqs. (2) with

$$
\begin{aligned}
& A_{+}(0, y, t)=0, \quad A_{+}(x, 0, t)=r A_{-}(x, 0, t), \\
& A_{-}(0, y, t)=0, \quad A_{-}\left(x, l_{y}, t\right)=r A_{+}\left(x, l_{y}, t\right), \\
& N_{+}(0, y, t)=1, \quad N_{-}(0, y, t)=1 \\
& A_{ \pm}(x, y, 0)=0, \quad N_{ \pm}(x, y, 0)=1 .
\end{aligned}
$$

'This system is representative of a rectangular constant amplitude laser pulse of semiinfinite extent in the negative $x$ direction, propagating to the right at speed $v_{0}$ where at $t=x=0$ its leading edge enters a plasma channel which is bounded by $0 \leq x \leq l_{x}$ and $0 \leq y \leq l_{y}$ and has a uniform Langmuir seed for the initiation of the instability. Within the channel the laser pulse decays into both an upgoing and downgoing electron plasma (Langmuir) wave and scattered light (Stokes) wave which propagates obliquely to the right $(0 \leq \theta \leq \pi / 2)$ and reflects off of the channel walls at $y=0$ and $y=l_{y}$ with a reflectivity coefficient $0 \leq r(\theta) \leq 1$. Note that at $x=0$ it is only necessary to define either the Stokes 
or Langmuir amplitude and the other condition becomes a consequence of the interaction. Additionally, it is the boundary conditions and not the governing equations that couple the upgoing and downgoing waves so that in the zero reflectivity limit we recover the interaction studied in Ref. 12 .

Using the characteristic variables $\xi=v_{1 y} x /\left(l_{y} v_{1 x}\right), \eta=y / l_{y}$ and $\tau=$ $v_{1 y}\left(v_{0} t-x\right) /\left[l_{y}\left(v_{0}-v_{1 x}\right)\right]$, and defining $B_{ \pm}=\left[\left(v_{0}-v_{1 x}\right) / v_{0}\right]^{1 / 2} A_{ \pm}$and $\gamma=$ $\gamma_{0} l_{y}\left[\left(v_{0}-v_{1 x}\right) / v_{0}\right]^{1 / 2} / v_{1 y}$, Eqs. (2) and (3) become

$$
\left(\partial_{\tau} \pm \partial_{\eta}+\partial_{\xi}\right) B_{ \pm}=\gamma N_{ \pm}, \quad \partial_{\tau} N_{ \pm}=\gamma B_{ \pm}
$$

and

$$
\begin{aligned}
& B_{+}(0, \eta, \tau)=0, \quad B_{+}(\xi, 0, \tau)=r B_{-}(\xi, 0, \tau) \\
& B_{-}(0, \eta, \tau)=0, \quad B_{-}(\xi, 1, \tau)=r B_{+}(\xi, 1, \tau) \\
& N_{+}(0, \eta, \tau)=1, \quad N_{-}(0, \eta, \tau)=1 \\
& B_{ \pm}(\xi, \eta, 0)=0, \quad N_{ \pm}(\xi, \eta, 0)=1
\end{aligned}
$$

within the plasma channel. If we first take the Laplace transform of Eqs. (4) and (5) with respect to $\tau$ (Laplace transform variable $s$ ) and then with respect to $\xi$ (Laplace transform variable $q$ ) we get

$$
\left( \pm \partial_{\eta}+s+q\right) \overline{\bar{B}}_{ \pm}=\gamma \overline{\bar{N}}_{ \pm}, \quad s \overline{\bar{N}}_{ \pm}-1 / q=\gamma \overline{\bar{B}}_{ \pm}
$$

and

$$
\begin{aligned}
& \overline{\bar{B}}_{+}(0, \eta, s)=0, \quad \overline{\bar{B}}_{+}(q, 0, s)=r \overline{\bar{B}}_{-}(q, 0, s) \\
& \overline{\bar{B}}_{-}(0, \eta, s)=0, \quad \overline{\bar{B}}_{-}(q, 1, s)=r \overline{\bar{B}}_{+}(q, 1, s) \\
& \overline{\bar{N}}_{+}(0, \eta, s)=1, \quad \overline{\bar{N}}_{-}(0, \eta, s)=1 \\
& \overline{\bar{B}}_{ \pm}(q, \eta, 0)=0, \quad \overline{\bar{N}}_{ \pm}(q, \eta, 0)=1 /(s q)
\end{aligned}
$$

where the double overbar denotes that a double Laplace transform has been taken. 'The- 
solutions to Eqs. (6) and (7) are

$$
\begin{aligned}
& \overline{\bar{B}}_{+}=\frac{1-r}{1-r e^{(\alpha-q)}} \frac{e^{-q \eta}}{q(\alpha-q)} \frac{\gamma}{s} e^{\alpha \eta}-\frac{1}{q(\alpha-q)} \frac{\gamma}{s} \\
& \overline{\bar{B}}_{-}=\frac{1-r}{1-r e^{(\alpha-q)}} \frac{e^{-q(1-\eta)}}{q(\alpha-q)} \frac{\gamma}{s} e^{\alpha(1-\eta)}-\frac{1}{q(\alpha-q)} \frac{\gamma}{s} \\
& \overline{\bar{N}}_{+}=\frac{1-r}{1-r e^{(\alpha-q)}} \frac{e^{-q \eta}}{q(\alpha-q)}\left(\frac{\gamma}{s}\right)^{2} e^{\alpha \eta}-\frac{1}{q(\alpha-q)}\left(\frac{\gamma}{s}\right)^{2}+\frac{1}{s q} \\
& \overline{\bar{N}}_{-}=\frac{1-r}{1-r e^{(\alpha-q)}} \frac{e^{-q(1-\eta)}}{q(\alpha-q)}\left(\frac{\gamma}{s}\right)^{2} e^{\alpha(1-\eta)}-\frac{1}{q(\alpha-q)}\left(\frac{\gamma}{s}\right)^{2}+\frac{1}{s q}
\end{aligned}
$$

where $\alpha=\gamma^{2} / s-s$. Close inspection of the amplitudes in Eqs. (8) shows that the lateral symmetry in the governing equations and initial and boundary conditions is manifested in such a way that the downgoing amplitudes are simply the mirror images of the upgoing amplitudes: $B_{-}(\xi, \eta, \tau)=B_{+}(\xi, 1-\eta, \tau)$ and $N_{-}(\xi, \eta, \tau)=N_{+}(\xi, 1-\eta, \tau)$. Furthermore, reflections are solely introduced in the solutions as $(1-r) /\left[1-r e^{(\alpha-q)}\right]$ coefficients in the first terms of the daughter wave amplitudes so that for the zero reflectivity case we recover the zero damping limit $\left(h_{1}=h_{2}=0\right)$ of Eqs. (8) of Ref. 12. These coefficients can be cast in a more useful form by applying $1 /(1-\beta)=\sum_{n=0}^{\infty} \beta^{n}$ to each coefficient. (The validity of employing this identity for our purposes is contingent on $\left|r e^{(\alpha-q)}\right|$ being less than unity and is discussed in detail in Refs. 13 and 24.) For inverse Laplace transforms with respect to $q$ we use

$$
\begin{aligned}
\mathcal{L}^{-1}\left[\frac{1}{q(\alpha-q)}\right] & =\frac{1-e^{\alpha \xi}}{\alpha}, \\
\mathcal{L}^{-1}\left[\overline{\bar{F}}(q) e^{-q \eta}\right] & =\bar{F}(\xi-\eta) I I(\xi-\eta),
\end{aligned}
$$


so that the inverse Laplace transform of Eqs. (8) can be cast in the form

$$
\begin{aligned}
\bar{B}_{+}= & \frac{\gamma}{\alpha s}+\frac{\gamma}{\alpha s} e^{\alpha \xi} H(\eta-\xi)+\frac{\gamma}{\alpha s} e^{\alpha \eta} H(\xi-\eta) \\
+ & \sum_{n=1}^{\infty} r^{n}\left[-\frac{\gamma}{\alpha s} e^{\alpha \xi} H(\xi-\eta-n)+\frac{\gamma}{\alpha s} e^{\alpha \xi} H(\xi-\eta-n+1)\right. \\
& \left.+\frac{\gamma}{\alpha s} e^{\alpha(\eta+n)} H(\xi-\eta-n)-\frac{\gamma}{\alpha s} e^{\alpha(\eta+n-1)} I I(\xi-\eta-n+1)\right], \\
\bar{N}_{+}= & -\frac{\gamma^{2}}{\alpha s^{2}}+\frac{1}{s}+\frac{\gamma^{2}}{\alpha s^{2}} e^{\alpha \xi} H(\eta-\xi)+\frac{\gamma^{2}}{\alpha s^{2}} e^{\alpha \eta} H(\xi-\eta) \\
+ & \sum_{n=1}^{\infty} r^{n}\left[-\frac{\gamma^{2}}{\alpha s^{2}} e^{\alpha \xi} H(\xi-\eta-n)+\frac{\gamma^{2}}{\alpha s^{2}} e^{\alpha \xi} H(\xi-\eta-n+1)\right. \\
& \left.+\frac{\gamma^{2}}{\alpha s^{2}} e^{\alpha(\eta+n)} H(\xi-\eta-n)-\frac{\gamma^{2}}{\alpha s^{2}} e^{\alpha(\eta+n-1)} H(\xi-\eta-n+1)\right],
\end{aligned}
$$

where $H$ is the Heaviside step function. The remaining nontrivial inverse I Laplace transforms are all of the form $\gamma e^{\alpha \psi} /(\alpha s)$ for the Stokes wave and $\gamma^{2} e^{\alpha \psi} /\left(\alpha s^{2}\right)$ for the Langmuir wave. These inverse transforms have been solved previously in Eqs. (7)-(15) of Ref. 8 and are the fundamental solutions of the governing equations [7, 8, 10-12]. Explicitly they are

$$
\begin{aligned}
& F_{S}(\psi, \tau)=\left[\sum_{m=0}^{\infty}\left(\frac{\psi}{\tau-\psi}\right)^{m+1 / 2} I_{2 m+1}\left\{2 \gamma[\psi(\tau-\psi)]^{1 / 2}\right\}-\sinh (\gamma \tau)\right] H(\tau-\psi) \\
& F_{L}(\psi, \tau)=\left[\sum_{m=0}^{\infty}\left(\frac{\psi}{\tau-\psi}\right)^{m} I_{2 m}\left\{2 \gamma[\psi(\tau-\psi)]^{1 / 2}\right\}-\cosh (\gamma \tau)\right] H(\tau-\psi)
\end{aligned}
$$

for the Stokes and Langmuir amplitudes respectively.

The upgoing daughter wave amplitudes for forward SRS in a two-dimensional - 
collisionless plasma channel are now evident given Eqs. (10) and (11):

$$
\begin{aligned}
B_{+}(\xi, \eta, \tau)= & \sinh (\gamma \tau)+F_{S}(\xi, \tau) H(\eta-\xi)+F_{S}(\eta, \tau) H(\xi-\eta) \\
& +\sum_{n=1}^{\infty} r^{n}\left[-F_{S}(\xi, \tau) H(\xi-\eta-n)+F_{S}(\xi, \tau) H(\xi-\eta-n+1)\right. \\
& \left.\quad+F_{S}(\eta+n, \tau) H(\xi-\eta-n)-F_{S}(\eta+n-1, \tau) I I(\xi-\eta-n+1)\right] \\
N_{+}(\xi, \eta, \tau)= & \cosh (\gamma \tau)+F_{L}(\xi, \tau) H(\eta-\xi)+F_{L}(\eta, \tau) H(\xi-\eta) \\
& +\sum_{n=1}^{\infty} r^{n}\left[-F_{L}(\xi, \tau) H(\xi-\eta-n)+F_{L}(\xi, \tau) H(\xi-\eta-n+1)\right. \\
& \left.+F_{L}(\eta+n, \tau) H(\xi-\eta-n)-F_{L}(\eta+n-1, \tau) H(\xi-\eta-n+1)\right]
\end{aligned}
$$

and the downgoing wave amplitudes are given by $B_{-}(\xi, \eta, \tau)=B_{+}(\xi, 1-\eta, \tau)$ and $N_{-}(\xi, \eta, \tau)=N_{+}(\xi, 1-\eta, \tau)$ where there is also the restriction that the amplitudes are zero outside of the plasma $(x<0)$ and have their initial values ahead of the laser pulse $\left(x>v_{0} t\right)$. Amplitudes outside of the channel and laser pulse but within the surrounding plasma $\left(y<0, y>l_{y}\right)$ are discussed in section IV of Ref. 12 .

We have purposefully written the amplitudes in Eqs. (12) as sums in $r^{n}$ to highlight the physical implication of reflections. In fact, all of the $r^{0}$ terms are equivalent to the undamped solutions studied in Ref. 12 (here they are merely expressed in terms of the fundamental solutions $F_{S, L}$ and the characteristic variables $\xi, \eta$, and $\tau$ ). Furthermore, each of the terms in the sum for $n$ greater than zero can be thought of as being the result of image sources outside of the plasma channel. Thus, the inclusion of reflections is equivalent to the addition of an infinite series of image sources. The effect of these image sources on the spatiotemporal evolution of the daughter waves within the channel is best shown by - 
rewriting Eqs. (12) as

$$
\begin{aligned}
B_{+}(\xi, \eta, \tau) & =\sinh (\gamma \tau) H(\xi-\tau) H(\eta-\tau) \\
+ & {\left[F_{S}(\xi, \tau)+\sinh (\gamma \tau)\right] H(\tau-\xi) H(\xi-\eta) } \\
& +\sum_{n=1}^{\infty} r^{n} \sinh (\gamma \tau) H(\xi-\tau) H(\tau-\eta+1-n) H(n-\tau+\eta) \\
& +\sum_{n=1}^{\infty} r^{n}\left[F_{S}(\xi, \tau)+\sinh (\gamma \tau)\right] H(\tau-\xi) H(\xi-\eta+1-n) H(n-\xi+\eta) \\
+ & (1-r) \sum_{n=0}^{\infty} r^{n}\left[F_{S}(\eta+n, \tau)+\sinh (\gamma \tau)\right] H(\tau-\eta-n) H(\xi-\eta-n) \\
N_{+}(\xi, \eta, \tau)= & \cosh (\gamma \tau) H(\xi-\tau) H(\eta-\tau) \\
+ & {\left[F_{L}(\xi, \tau)+\cosh (\gamma \tau)\right] H(\tau-\xi) H(\xi-\eta) } \\
& +\sum_{n=1}^{\infty} r^{n} \cosh (\gamma \tau) H(\xi-\tau) H(\tau-\eta+1-n) H(n-\tau+\eta) \\
& +\sum_{n=1}^{\infty} r^{n}\left[F_{L}(\xi, \tau)+\cosh (\gamma \tau)\right] H(\tau-\xi) H(\xi-\eta+1-n) H(n-\xi+\eta) \\
& +(1-r) \sum_{n=0}^{\infty} r^{n}\left[F_{L}(\eta+n, \tau)+\cosh (\gamma \tau)\right] H(\tau-\eta-n) H(\xi-\eta-n)
\end{aligned}
$$

In the nonreflective theory of Ref. 12 we had three distinct regions of spatiotemporal growth. In the reflective theory, we will now have three distinct regions of growth for each $n$ in Eqs. (13) (Fig. 2). For $n=0$ we will have the same three regions as in the nonreflective theory with the two-dimensional amplitudes multiplied by a factor of $(1-r)$. In terms of the space and time variables $x, y$, and $t$, the first region for $n \geq 1$ is $v_{1 x}\left[y+(n-1) l_{y}\right] / v_{1 y} v_{1 x}\left(y+n l_{y}\right) / v_{1 y}<x \leq v_{1 x}\left(y+n l_{y}\right) / v_{1 y} \leq$ and $x<v_{1 x} t$ and will contribute a one-dimensional sum of Bessel functions multiplied by $r^{n}$; the second region is $v_{1 x}\left(y+n l_{y}\right) / v_{1 y}<x<v_{0} t-\left(v_{0}-v_{1 x}\right)\left(y+n l_{y}\right) / v_{1 y}$ and will contribute a twodimensional sum of Bessel functions multiplied by $(1-r) r^{n}$; the third region is $x \geq v_{1 x} t$ and $v_{0} t-\left(v_{0}-v_{1 x}\right)\left(y+n l_{y}\right) / v_{1 y} \leq x<v_{0} t-\left(v_{0}-v_{1 x}\right)\left[y+(n-1) l_{y}\right] / v_{1 y}$ and will contribute a one-dimensional hyperbolic function multiplied by $r^{n}$. The representation of these regions- 
in Fig. 2 shows that the spatiotemporal character of each reflection is manifested in such a way that the total spatiotemporal character of the amplitudes is unchanged in that for any finite number of reflections there are only three regions of general spatiotemporal growth for the total amplitude (distinguished by the solid lines in Fig. 2). Region I is characterized by one-dimensional Bessel growth, region II is characterized by one and two-dimensional Bessel growth and one-dimensional exponential (hyperbolic) growth, and region III is characterized purely by exponential (hyperbolic) growth. Comparing these regions with that of Ref. 12 shows that the inclusion of a finite number of reflections does not alter the general spatiotemporal character between the reflective and nonreflective theories, but it does significantly change the daughter wave amplitudes within each corresponding $n$th subregion of region II.

Plots of Eqs. (12) and (13) can be found in Fig. 3. In each plot a snapshot of the upgoing Langmuir amplitude is shown for a given time and forward scattering angle where the scattering angle of the Stokes wave is indicated in the top center, the solid lines distinguish the three distinct general regions of spatiotemporal growth, and each plot is normalized so that the largest amplitude is white and smallest amplitude is dark gray. The chosen time is $t=1$ so that the leading edge of the laser pulse is at $x=v_{0} t=1$ and the plasma is bounded by $0 \leq x \leq 1$ and $0 \leq y \leq 0.5$ with reflective boundaries at $y=0$ and $y=0.5$ with a reflection coefficient of $r=0.5$. All of the other plasma parameters are the same as in Fig. 3 of Ref. 12 so that direct comparisons can be made between the reflective and nonreflective theories. It is immediately obvious from Fig. 3 that the inclusion of a finite number of reflections does not alter the dividing lines between the three general regions of growth. It is also immediately obvious that the amplitudes in region I and III are exactly the same as in the nonreflective theory and there is a vast difference in the spatiotemporal evolution of region II in the reflective and nonreflective theories. 'The reflective theory predicts amplitudes in region 11 that are greater than the nonreflective theory and depend less on the lateral spatial variable $y$. This can be attributed to the- 
manner in which each of the $n$ reflections in Eqs. (13) contribute to the total amplitude. From Fig. 2 we see that each $n$th reflection has its own three regions of growth, and each $n$-plus-first reflection adds in such a way that its regions I and III overlap the $n$th region II. Sincc regions I and III are both one dimensional in nature and region II is two-dimensional we see that for a finitely large number of reflections the total amplitude in the general two-dimensional region can become dominated by one-dimensional growth.

If we now consider damping within the plasma channel then the governing equations become

$$
\left(\partial_{t} \pm v_{1 y} \partial_{y}+v_{1 x} \partial_{x}+\nu_{1}\right) A_{ \pm}=\gamma_{0} N_{ \pm}, \quad\left(\partial_{t}+\nu_{2}\right) N_{ \pm}=\gamma_{0} A_{ \pm}
$$

along with the boundary and initial conditions of Eqs. (3). It is not difficult to show that the solutions for these governing equations are given by Eqs. (12) and (13) and all of the above arguments and discussions are valid if we simply replace the fundamental solutions [Eqs. (11)] with their damped counterparts [12]:

$$
\begin{aligned}
F_{S}(\psi, \tau) & =H(\tau-\psi)\left[-\gamma p e^{-h_{s} \tau} \sinh (\tau / p)+\gamma p e^{-h_{2} \tau} e^{-2 h_{d} \psi}\right. \\
& \left.\times \sum_{m=1}^{\infty} \frac{\left(h_{d} p+1\right)^{m}-\left(h_{d} p-1\right)^{m}}{2(\gamma p)^{m}}\left(\frac{\psi}{\tau-\psi}\right)^{m / 2} I_{m}\left\{2 \gamma[\psi(\tau-\psi)]^{1 / 2}\right\}\right] \\
F_{L}(\psi, \tau) & =H(\tau-\psi)\left[-h_{d} p e^{-h_{s} \tau} \sinh (\tau / p)+e^{-h_{s} \tau} \cosh (\tau / p)+\gamma p e^{-h_{2} \tau} e^{-2 h_{d} \psi}\right. \\
& \left.\times \sum_{m=1}^{\infty} \frac{\left(h_{d} p+1\right)^{m}-\left(h_{d} p-1\right)^{m}}{2(\gamma p)^{m}}\left(\frac{\psi}{\tau-\psi}\right)^{(m-1) / 2} I_{m-1}\left\{2 \gamma[\psi(\tau-\psi)]^{1 / 2}\right\}\right]
\end{aligned}
$$

where $h_{1}=\nu_{1} l_{y} / v_{1 y}, h_{2}=\nu_{2} l_{y}\left(v_{0}-v_{1 x}\right) /\left(v_{0} v_{1 y}\right), h_{s}=\left(h_{1}+h_{2}\right) / 2, h_{d}=\left(h_{1}-h_{2}\right) / 2$, and $p=\left(h_{d}^{2}+\gamma^{2}\right)^{-1 / 2}$.

\section{TWO-DIMENSIONAL BACKWARD SRS}

The governing equations and boundary and initial conditions for two-dimensional backward SRS in a plasma channel are given by

$$
\left(\partial_{t} \pm v_{1 y} \partial_{y}-v_{1 x} \partial_{x}\right) A_{ \pm}=\gamma_{0} N_{ \pm}, \quad \partial_{t} N_{ \pm}=\gamma_{0} A_{ \pm}
$$




$$
\begin{aligned}
& A_{+}\left(l_{x}, y, t\right)=0, \quad A_{+}(x, 0, t)=r A_{-}(x, 0, t), \\
& A_{-}\left(l_{x}, y, t\right)=0, \quad A_{-}\left(x, l_{y}, t\right)=r A_{+}\left(x, l_{y}, t\right), \\
& N_{+}\left(l_{x}, y, t\right)=1, \quad N_{-}\left(l_{x}, y, t\right)=1, \\
& A_{ \pm}(x, y, 0)=0, \quad N_{ \pm}(x, y, 0)=1,
\end{aligned}
$$

where $v_{1 x}=\left|\mathbf{v}_{1 x}\right|$ so that the Stokes wave is now propagating obliquely to the left $(\pi / 2 \leq$ $\theta \leq \pi)$. The geometry of the interaction is the same as in the two-dimensional forward case with the laser pulse being of semi-infinite extent in the negative $x$ direction and the plasma channel being bounded by $0 \leq x \leq l_{x}$ and $0 \leq y \leq l_{y}$. Note that at $x=0$ it is only necessary to define either the Stokes or Langmuir amplitude and the other condition becomes a consequence of the interaction. Additionally, it is the boundary conditions and not the governing equations that couple the upgoing and downgoing waves so that in the zero reflectivity limit we recover the backward interaction studied in Ref. 12 .

Using the characteristic variables $\xi=v_{1 y}\left(l_{x}-x\right) /\left(l_{y} v_{1 x}\right), \eta=y / l_{y}$, and $\tau=v_{1 y}\left(v_{0} t-x\right) /\left[l_{y}\left(v_{0}+v_{1 x}\right)\right]$, and defining $B_{ \pm}=\left[\left(v_{0}+v_{1 x}\right) / v_{0}\right]^{1 / 2} A_{ \pm}$and $\gamma=$ $\gamma_{0} l_{y}\left[\left(v_{0}+v_{1 x}\right) / v_{0}\right]^{1 / 2} / v_{1 y}$, Eqs. (16) and (17) for the region within the plasma channel $\left(0 \leq x \leq l_{x}\right.$ and $\left.0 \leq y \leq l_{y}\right)$ will have the same form as Eqs. (4) and (5). Hence, the daughter wave amplitudes for backward SRS in a two-dimensional plasma channel have the same form as those given by Eqs. (11) and (12) with the proper characteristic variables and definitions $(\xi, \eta, \tau$, and $\gamma)$ associated with the backward interaction.

Due to the symmetry between the forward and backward interactions, the discussion of image sources and the spatiotemporal evolution of the daughter amplitudes in the previous section is still valid for backward scattering. However, in terms of the space and time variables $x, y$, and $t$ the relevant space-time regions (Fig. 4) for $n \geq 1$ are region $\mathrm{I}$ in which $x<v_{0} t-\left(v_{0}+v_{1 x}\right)\left(y+n l_{y}\right) / v_{1 y}$ and $x \leq l_{x}-v_{1 x}\left(y+n l_{y}\right) / v_{1 y}$ which will contribute a two-dimensional sum of Bessel functions multiplied by $(1-r) r^{n}$, region II in which $x \leq\left(v_{0}+v_{1 x}\right) l_{x} / v_{0}-v_{1 x} t$ and $v_{0} t-\left(v_{0}+v_{1 x}\right)\left(y+n l_{y}\right) / v_{1 y} \leq$ $x<v_{0} t-\left(v_{0}+v_{1 x}\right)\left[y+(n-1) l_{y}\right] / v_{1 y}$ which will contribute a one-dimensional hyper-- 
bolic function multiplied by $r^{n}$, region III in which $x>\left(v_{0}+v_{1 x}\right) l_{x} / v_{0}-v_{1 x} t$ and $l_{x}-v_{1 x}\left(y+n l_{y}\right) / v_{1 y} \leq x<l_{x}-v_{1 x}\left[y+(n-1) l_{y}\right] / v_{1 y}$ which will contribute a onedimensional sum of Bessel functions multiplied by $r^{n}$, and there is also the restriction that the amplitudes are zero outside of the plasma $(x<0)$ and have their initial values ahead of the laser pulse $\left(x>v_{0} t\right)$. Amplitudes outside of the channel and laser pulse but within the surrounding plasma $\left(y<0, y>l_{y}\right)$ are discussed in section IV of Ref. 12.

Plots of Eqs. (12) and (13) for backward scattering can be found in Fig. 5. In each plot a snapshot of the upgoing Langmuir amplitude is shown for a given time and backward scattering angle where the scattering angle of the Stokes wave is indicated in the top center, the solid lines distinguish the three distinct general regions of spatiotemporal growth, and each plot is normalized so that the largest amplitude is white and smallest amplitude is dark gray. The chosen time is $l=1.25$ so that the leading edge of the laser pulse is at $x=v_{0} t=1.25$ (outside of the plasma) and the plasma is bounded by $0 \leq x \leq 1$ and $0 \leq y \leq 0.5$ with reflective boundaries at $y=0$ and $y=0.5$ with a reflection coefficient of $r=0.5$. All of the other plasma parameters are the same as in Fig. 6 of Ref. 12 so that direct comparisons can be made between the reflective and nonreflective theories. As in the forward scattering case it is obvious from Fig. 5 that the inclusion of a finite number of reflections does not alter the dividing lines between the three general regions of growth. It is also obvious that the amplitudes in region II are greater than that predicted by the nonreflective theory and depend less on the lateral spatial variable $y$. As in the forward case, this structure is due to the overlapping of the $n$th two-dimensional region by the $n$-plus-first one dimensional regions (Fig. 4).

If we now consider damping within the plasma channel then the governing equations for the backward interaction become

$$
\left(\partial_{t} \pm v_{1 y} \partial_{y}-v_{1 x} \partial_{x}+\nu_{1}\right) A_{ \pm}=\gamma_{0} N_{ \pm}, \quad\left(\partial_{t}+\nu_{2}\right) N_{ \pm}=\gamma_{0} A_{ \pm}
$$

along with the boundary and initial conditions of Eqs. (17). It is not difficult to show that the solutions for these governing equations are given by Eqs. (12)- 
and (13) with the damped fundamental solutions given by Eqs. (15) where $h_{1}=$ $\nu_{1} l_{y} / v_{1 y}, h_{2}=\nu_{2} l_{y}\left(v_{0}+v_{1 x}\right) /\left(v_{0} v_{1 y}\right), h_{s}=\left(h_{1}+h_{2}\right) / 2, h_{d}=\left(h_{1}-h_{2}\right) / 2, \gamma=$ $\gamma_{0} l_{y}\left[\left(v_{0}+v_{1 x}\right) / v_{0}\right]^{1 / 2} / v_{1 y}, p=\left(h_{d}^{2}+\gamma^{2}\right)^{-1 / 2}$ and the variables $\xi, \eta$, and $\tau$ are those associated with the backward interaction.

\section{DISCUSSION OF THE ZERO TRANSMISSION CASE}

To determine the daughter wave amplitudes when there is total reflectivity $(r-1)$ we can simply substitute $r-1$ into Eqs. (13) and we are left with

$$
\begin{aligned}
& B_{+}(\xi, \eta, \tau)=\sinh (\gamma \tau) H(\xi-\tau)+\left[F_{S}(\xi, \tau)+\sinh (\gamma \tau)\right] H(\tau-\xi), \\
& N_{+}(\xi, \eta, \tau)=\cosh (\gamma \tau) H(\xi-\tau)+\left[F_{L}(\xi, \tau)+\cosh (\gamma \tau)\right] H(\tau-\xi),
\end{aligned}
$$

which has no dependence on the lateral spatial coordinate $(\eta)$ so that the upgoing amplitudes are identical to the downgoing amplitudes. Equations (19) are identical to Eqs. (A26) of Ref. 10 implying that the daughter wave amplitudes for two-dimensional oblique scattering within a zero transmission (totally reflecting) plasma channel are equivalent to the one-dimensional amplitudes derived for the case of laterally transmitting plasma channel.

In this light the one-dimensional amplitudes derived in Ref. 10 can be interpreted as representing the solutions for direct forward SRS where the daughter Stokes wave propagates forward with a velocity $v_{1}=v_{1 x}$. Since for that case both the daughter wave and the pump wave are propagating in the direct forward direction no energy is lost in the transverse direction. For the current case (a two-dimensional interaction propagating in a plasma channel with reflectivity coefficient of one) we have the Stokes wave propagating obliquely but with a forward velocity of $v_{1 x}$. For $r=1$ the channel walls are totally reflecting so there is also no loss of energy in the transverse direction and the two scenarios are equivalent. (For further discussion on the role of these one-dimensional amplitudes in two-dimensional scattering processes see Ref. 12.) Furthermore, Eqs. (19) are also the solutions for direct forward scattering in the plasma channel for any reflectivity coefficient since direct forward scattering is not altered by the inclusion of lateral reflections. 'Ihus' 
we have created a scenario analogous to that of traveling waves in a perfectly reflecting waveguide, that is, oblique reflections with a forward velocity of $v_{1 x}$ in a perfectly reflecting channel are equivalent to direct forward scattering with a velocity of $v_{1 x}$ (within or without a channel). Note that all of these arguments are also valid for the case of backward scattering and those amplitudes are given by Eqs. (29) of Ref. 10 with the replacement $v_{1}=v_{1 x}$.

\section{DISCUSSION OF THE REFLECTIVITY COEFFICIENT}

The daughter wave amplitudes in sections II and III are valid for any reflectivity coefficient $(0 \leq r(\theta) \leq 1)$ that is a function of the scattering angle. The most simple model consists of a sharply bounded plasma channel of density $n_{1} / n_{c}$ surrounded by plasma with a density of $n_{2} / n_{c}$ where $n_{c}$ is the critical density [Fig. 6(a)]. Using standard techniques the reflectivity coefficient for this model is given by:

$$
r\left(\theta_{S}\right)=\left|\frac{\delta_{1} \cos \theta_{I}-\delta_{2} \cos \theta_{T}}{\delta_{1} \cos \theta_{I}+\delta_{2} \cos \theta_{T}}\right|
$$

where the $I$ and $T$ subscripts indicate the incident and transmitted angles which are related by:

$$
\cos \theta_{T}=\left[1-\left(\frac{\delta_{1}}{\delta_{2}} \sin \theta_{I}\right)^{2}\right]^{1 / 2}
$$

where the indices of refraction are $\delta_{i}=\left(1-n_{i} / n_{c}\right)^{1 / 2}$. With Fig. $6(\mathrm{~b})$ the reflectivity coefficient can be interpreted as a function of the Stokes scattering angle and is plotted in Fig. 7 for a plasma channel of density $n_{1}=0.01 n_{c}$ with walls of density $n_{2}=0.1 n_{c}$ and $0.3 n_{c}$. More complicated channel density profiles such as those described in Refs. 25 and 26 can easily be incorporated into the model described herein by simply inserting the desired reflectivity coefficient into the expressions discussed in sections II and III.

\section{ASYMPTOTIC SOLUTIONS}

Detailed discussion of this section can be found in Refs. 23 and 24. Only a summary of the mathematics is included here. 
Time asymptotic amplitudes can be derived by using the inversion formula,

$$
F(x)=\frac{1}{2 \pi i} \int_{c-i \infty}^{c+i \infty} e^{q x} f(q) d q
$$

on the expressions in Eqs. (8). The integrands have singularities at $q=\alpha, 0$, and $q_{n}=$ $\alpha-\nu+2 \pi$ in where $\nu=-\ln r$. The singularities at $q=\alpha$ will yield no net contribution to the total amplitude and the singularities at $q=0$ have been discussed in Ref. (11). For the remaining singularities the contributions to the daughter wave amplitudes are all on the order of $e^{\alpha-\nu}$. Therefore

$$
\begin{aligned}
& \bar{B}_{+} \sim \frac{(1-r) e^{\nu \eta \eta}}{\nu}\left(\frac{\gamma}{s}\right) \frac{e^{(\alpha-\nu) \xi}}{(\alpha-\nu)}+\operatorname{Res}[q=0], \\
& \bar{N}_{+} \sim \frac{(1-r) e^{\nu \eta}}{\nu}\left(\frac{\gamma}{s}\right)^{2} \frac{e^{(\alpha-\nu) \xi}}{(\alpha-\nu)}+\operatorname{Res}[q=0],
\end{aligned}
$$

and the downgoing wave amplitudes are given by $\bar{B}_{-}(\xi, \eta, s)=\bar{B}_{+}(\xi, 1-\eta, s)$ and $\bar{N}_{-}(\xi, \eta, s)=\bar{N}_{+}(\xi, 1-\eta, s)$. The first term of each of these expressions is merely the the temporal Laplace transform of the fundamental damped solutions of the onedimensional governing equations [Eqs. (15) with $h_{1}=\nu$ and $h_{2}=0$ ] multiplied by a factor of $(1-r) e^{\nu \eta} / \nu$. Each of these fundamental solutions consists of a hyperbolic function subtracted from a sum of Bessel functions in the region $\xi<\tau$ and is zero for $\tau \leq \xi$. Reference 11 shows that for large times the contribution from the residue at $q=0$ is precisely the same as that for the hyperbolic part of the contribution from $q=\alpha-\nu$ but with opposite sign and its contribution is valid for both $\xi<\tau$ and $\tau \leq \xi$. Summing the dominant contributions from all of the singularities we find that the time asymptotic solutions for oblique scattering in a plasma channel of reflectivity $r$ are equivalent to the zero reflectivity solutions of the one-dimensional damped equations with $h_{1}=\nu$ and $h_{2}=0$ and multiplied by a factor of $(1-r) e^{\nu \eta} / \nu$. (For more on the one-dimensional damped solutions see Ref. 12.) 'I'hus, for large times (many reflections), one-dimensional amplitudes dominate the plasma (see the discussion of Figs. 2 and 4) and the dissipative loss created by the transmission of the Stokes wave through the channel boundaries is equivalent to an overalt- 
damping of the Stokes amplitude (Fig. 8). Note that if we are considering damping within the plasma channel $\left[\nu_{1} \neq 0\right.$ in Eqs. (14) and (18)] then the above still holds true and time asymptotically the overall damping is $\nu_{1}+\nu v_{1 y} / l_{y}$ for the Stokes wave.

\section{DISCUSSION OF THE TWO-DIMENSIONAL EVOLUTION}

Figure 9 shows comparisons between the one-dimensional nonreflective theory of Ref. 10 (lines with small dashes), the two-dimensional nonreflective theory of Ref. 12 (lines with large dashes) and the two-dimensional reflective theory developed herein (solid lines). Figure 9 is constructed with the same plasma parameters as in Figs. 3 and 5. Figure (a) is at time $t=1$ with a reflectivity coefficient of $r=0.5$, figure (b) is also at time $t=1$ but with a reflectivity coefficient given by Eq. (20) with $n_{1}=0.01 n_{c}$ and $n_{2}=0.1 n_{c}$, and figure (c) is at time $t=5$ and has the same reflectivity coefficient as in figure (b). It is evident from these figures that the two-dimensional reflective theory always predicts equal or larger gain than the two-dimensional nonreflective theory [a factor of 10 more in Fig. 9 (c)] and smaller or equivalent gain than the one-dimensional nonreflective theory [a factor of 10,000 less in Fig. 9 (c)]. When the reflection coefficient is zero [or close to zero as in Fig. 9 (b)] the two-dimensional reflective theory herein is equivalent to the nonreflective two-dimensional theory of Ref. 12 and the solid lines in Fig. 9 will be collinear with the lines with large dashes. When the reflection coefficient is one the two-dimensional reflective theory herein is equivalent to the nonreflective one-dimensional theory of Ref. 10 and the solid lines in Fig. 9 will be collinear with the lines with small dashes. For intermediate reflectivity coefficients the growth of the daughter waves is reliant on competition between the reflectivity coefficient and the aspect ratio of the laser pulse in such a way that for late times (many reflections) the complicated two-dimensional model of growth within the channel is equivalent to a much less complex one-dimensional damped model with zero channel reflectivity.

\section{SUMMARY}

In this paper we have developed an analytic model of laser driven parametric instabilities- 
within a plasma channel. Included are the effects of two spatial dimensions, damping, finite pulse and plasma boundaries, and oblique reflections of the daughter waves induced by lateral density variations. We have found that parametric instability growth within a channel is analogous to the propagation of electromagnetic waves in a leaky waveguide. Exact analytic solutions of the governing equations were found to be representative of an infinite series of image sources outside of the plasma channel which trap Stokes radiation and enhance instability growth within the channel through a transition from subdominant two-dimensional modes to dominant faster growing one-dimensional modes. In addition late time analysis showed that the partial transmission of Stokes radiation was in essence equivalent to an overall damping of the Stokes amplitude within the channel.

\section{ACKNOWLEDGMENTS}

This work was supported by the National Science Foundation under Contract No. PHY9415583, the United States Department of Energy (DOE) Office of Inertial Confinement Fusion under Cooperative Agreement No. DE-FC03-92SF19460, the University of Rochester, the New York State Energy Research and Development Authority, and the United States Department of Energy via the University of California Lawrence Livermore National Laboratory under contract No. W-7405-Eng-48. The support of DOE does not constitute an endorsement by DOE of the views expressed in this article. 


\section{REFERENCES}

[1] A. Bers, in Handbook of Plasma Physics, edited by R. Z. Sagdeev and M. N. Rosenbluth, Vol 1: Basic Plasma Physics, edited by A. A. Galeev and R. N. Sudan (North-Holland, Amsterdam, 1983), p. 451 and references therein.

[2] C. J. McKinstrie and R. Bingham, Phys. Fluids B 4, 2626 (1992).

[3] T. M. Antonsen, Jr. and P. Mora, Phys. Rev. Lett. 69, 2204 (1992).

[4] T. M. Antonsen, Jr. and P. Mora, Phys. Fluids B 5, 1440 (1993).

[5] W. B. Mori, C. D. Decker, D. E. Hinkel and T. Katsouleas, Phys. Rev. Lett. 72, 1482 (1994).

[6] S. C. Wilks, W. L. Kruer, E. A. Williams, P. Amendt and D. C. Eder, Phys. Plasmas 2, 274 (1995).

[7] C. J. McKinstrie, R. Betti, R. E. Giacone. T. Kolber and E. J. Turano, Phys. Rev. E 51, 3752 (1995).

[8] See National Technical Information Service document No. DOE/SF/19460-121 (E. J. Turano, C. J. McKinstrie and R. E. Giacone, "One-dimensional stimulated Raman scattering of short laser pulses," Laboratory for Laser Energetics Report 267, 1995.) Copies may be obtained from the National Technical Information Service, Springfield, VA 22161.

[9] C. D. Decker, W. B. Mori, T. Katsouleas and D. E. Hinkel, Phys. Plasmas 3, 1360 (1996).

[10] C. J. McKinstrie and E. J. Turano, Phys. Plasmas 3, 4683 (1996).

[11] C. J. McKinstrie, A. V. Kanaev, E. J. Turano, Phys. Rev. E

[12] C. J. McKinstrie and E. J. Turano, Phys. Plasmas 4, 3347 (1997).

[13] R. W. Boyd, K. Rzążewski, P. Narum, Phys. Rev. A 42, 5514 (1990).

[14] E. A. Williams and R. R. McGowan, in Research Trends in Physics: Inertial Confinement Fusion, edited by K. A. Brueckner (American Institute of Physics, New York, 1992), p. 325. 
[15] P. Mounaix, D. Pesme, W. Rozmus and M. Casanova, Phys. Fluids B 5, 3304 (1993).

[16] P. Mounaix and D. Pesme, Phys. Plasmas 1, 2579 (1994).

[17] C. J. McKinstrie, R. Betti, R. E. Giacone, T. Kolber and J. S. Li, Phys. Rev. E 50, 2182 (1994).

[18] D. E. Hinkel, E. A. Williams and R. L. Berger, Phys. Plasmas 1, 2987 (1994).

[19] D. E. Hinkel, E. A. Williams and R. L. Berger, Phys. Plasmas 2, 3447 (1995).

[20] R. E. Giacone, C. J. McKinstrie and R. Betti, Phys. Plasmas 2, 4596 (1995).

[21] C. J. McKinstrie, J. S. Li, R. E. Giacone and H. X. Vu, Phys. Plasmas 3, 2686 (1996).

[22] W. L. Kruer, The Physics of Laser Plasma Interactions (Addison-Wesley, Redwood City, California, 1988), Chap. 7.

[23] E. J. Turano, C. J. McKinstrie, and A. V. Kanaev, Bull. Am. Phys. Soc. 42, 1839 (1997).

[24] E. J. Turano, "Spatiotemporal evolution of Stimulated Raman Scattering Driven by Short Laser Pulses", Ph. D. Thesis, Department of Physics and Astronomy, University of Rochester, Rochester, New York, 14627 (1998).

[25] V. L. Ginzburg, Propagation of Elcctromagnctic waves in Plasma (Gordon and Breach, New York, New York, 1961).

[26] K. G. Budden, Radio Waves in the Ionosphere (Cambridge University Press, Cambridge, Great Britain, 1961). 


\section{FIGURE CAPTIONS}

Fig. 1 The geometry of SRS is shown in the laboratory frame for (a) the upgoing interaction and (b) the downgoing interaction. The range of scattering angles considered for the Stokes wave is $0 \leq \theta \leq \pi / 2$ for forward angles and $\pi / 2 \leq \theta \leq \pi$ for backward angles.

Fig. 2 The three distinct forward scattering regions of growth for each $n$ in Eqs. (13) are divided by the dashed lines. The three distinct general regions of forward scattering growth are divided by the solid lines (which are collinear with the $n=0$ dashed lines). The finite size of the channel is explicitly included in (b).

Fig. 3 Linearly shaded contour plots of the Langmuir amplitude [Eqs. (12) and (13)] are shown at time $t=1$ for three forward scattering angles. The solid lines distinguish the three general regions of growth. Each plot is normalized so that the largest amplitude is white and smallest amplitude is dark gray.

Fig. 4 The three distinct backward scattering regions of growth for each $n$ in Eqs. (13) are divided by the dashed lines. The three distinct general regions of backward scattering growth are divided by the solid lines (which are collinear with the $n=0$ dashed lines). The finite size of the channel is explicitly included in (b).

Fig. 5 Linearly shaded contour plots of the Langmuir amplitude [Eqs. (12) and (13)] are shown at time $t=1.25$ for two backward scattering angles. The solid lines distinguish the three general regions of growth. Each plot is normalized so that the largest amplitude is white and smallest amplitude is dark gray.

Fig. 6 (a) A basic model for the plasma channel density profile. (b) The scattering geometry at the plasma channel density gradient.

Fig. 7 The reflectivity coefficient [Eq. (20)] is plotted as a function of the Stokes scattering angle for a channel density of $0.01 n_{c}$ and surrounding plasma density of $0.1 n_{\mathrm{c}}$ and $0.3 n_{\mathrm{c}}$.

Fig. 8 The exact solution (solid lines) from Eqs. (12) and (13) is compared with the- 
time asymptotic solution (dashed lines) from the one-dimensional damped theory of Ref. 12 for (a) $t=1$, (b) $t=3$, and (c) $t=5$. The plasma parameters are the same as in Figs. 3 and 5.

Fig. 9 Comparison of the one-dimensional nonreflective theory of Ref. 10 (lines with small dashes), the two-dimensional nonreffective theory of Ref. 12 (lines with large dashes), and the two-dimensional reflective theory developed herein (solid lines). The temporal length of the interaction and reflectivity coefficients are given by: (a) $t=1, \mathrm{r}=0.5$, (b) $t=1, r$ given by Eq. (20) with $n_{1}=0.01 n_{c}$ and $n_{2}=0.1$, and (c) $t=5$ with the same reflectivity coefficient as (b). 

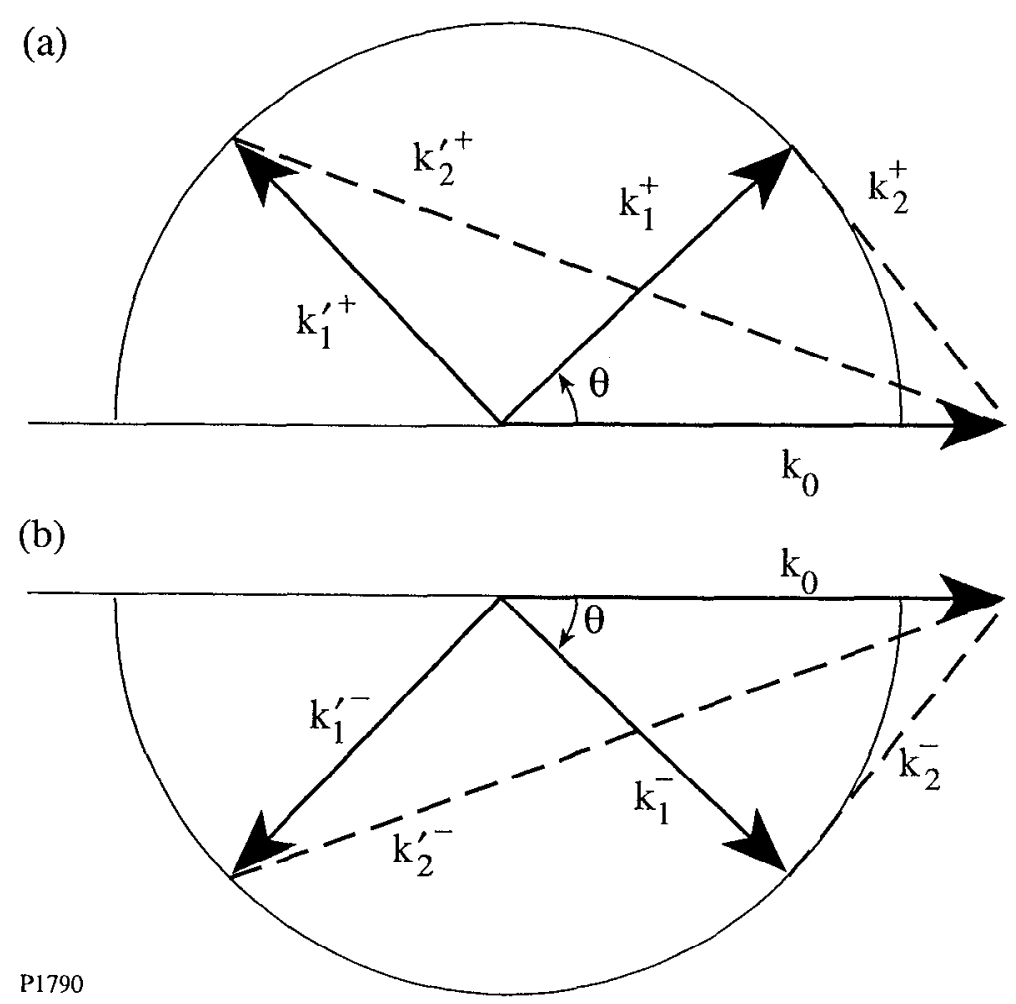

Fig. 1 The geometry of SRS is shown in the laboratory frame for (a) the upgoing interaction and (b) the downgoing interaction. The range of scattering angles considered for the Stokes wave is $0 \leq \theta \leq \pi / 2$ for forward angles and $\pi / 2 \leq \theta \leq \pi$ for backward angles. 


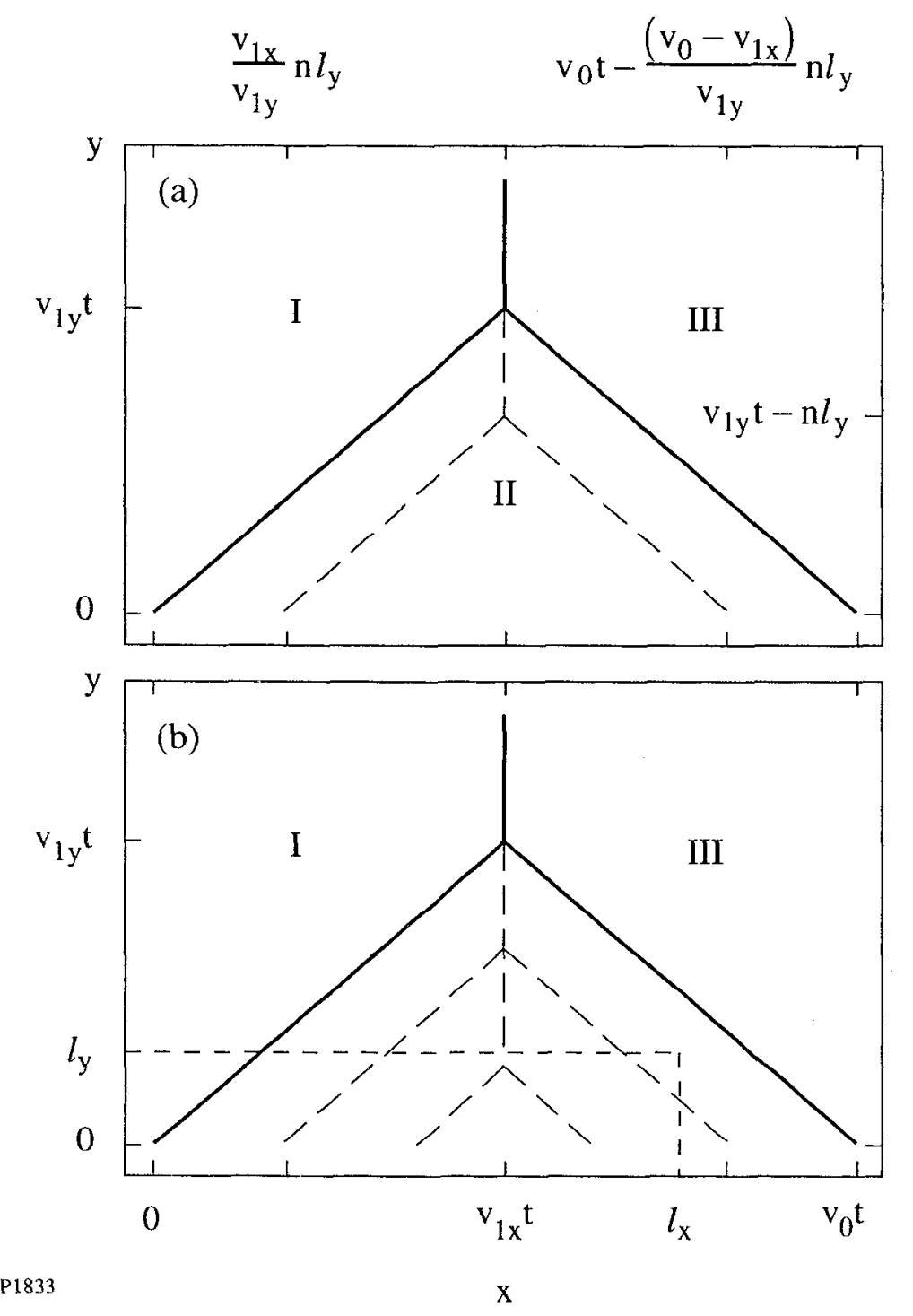

Fig. 2 The three distinct forward scattering rcgions of growth for each $n$ in Eqs. (13) are divided by the dashed lines. The three distinct general regions of forward scattering growth are divided by the solid lines (which are collinear with the $n=0$ dashed lines). The finite size of the channel is explicitly included in (b). 

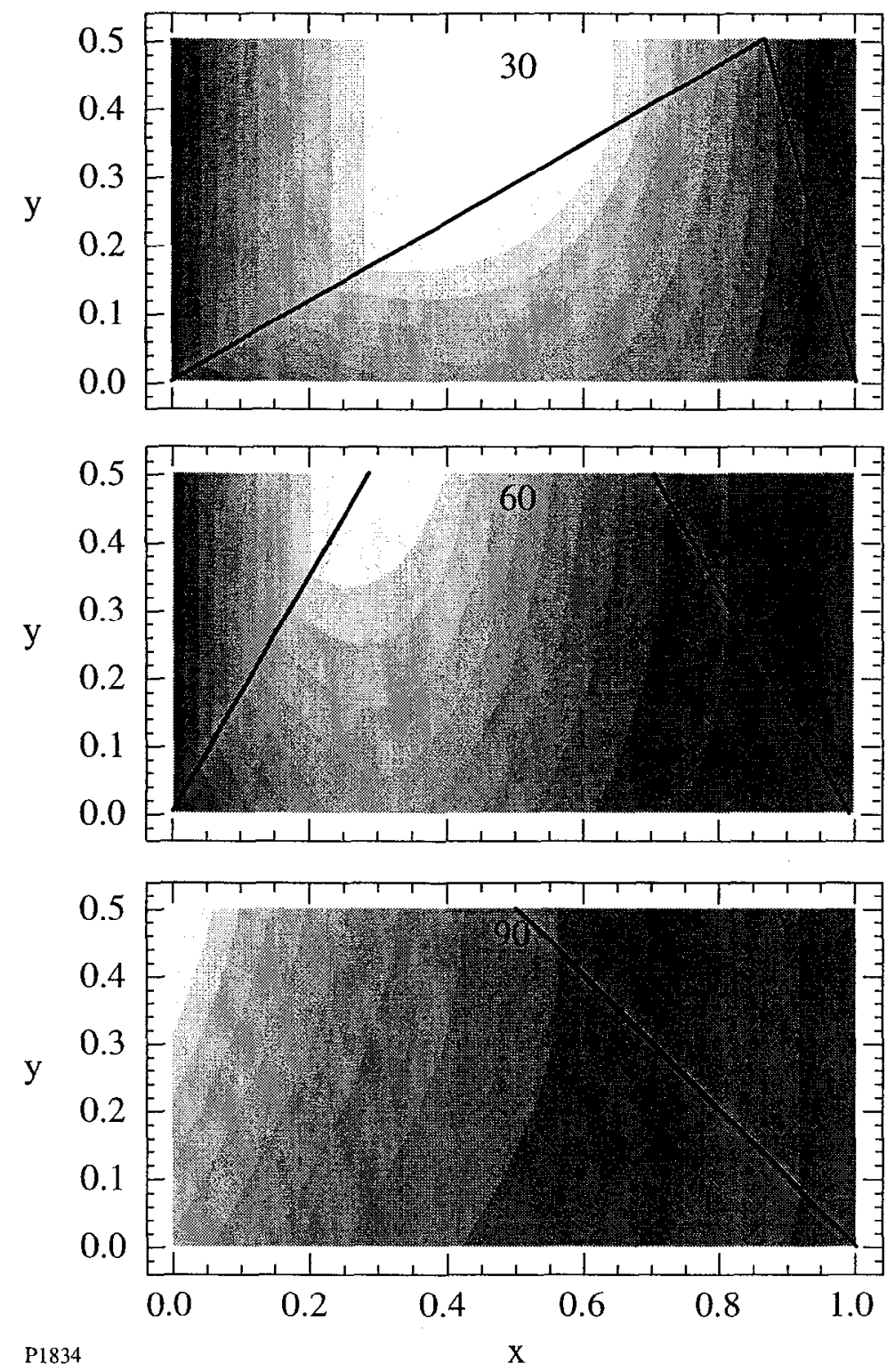

Fig. 3 Linearly shaded contour plots of the Langmuir amplitude [Eqs. (12) and (13)] are shown at time $t=1$ for three forward scattering angles. The solid lines distinguish the three general regions of growth. Each plot is normalized so that the largest amplitude is white and smallest amplitude is dark gray. 

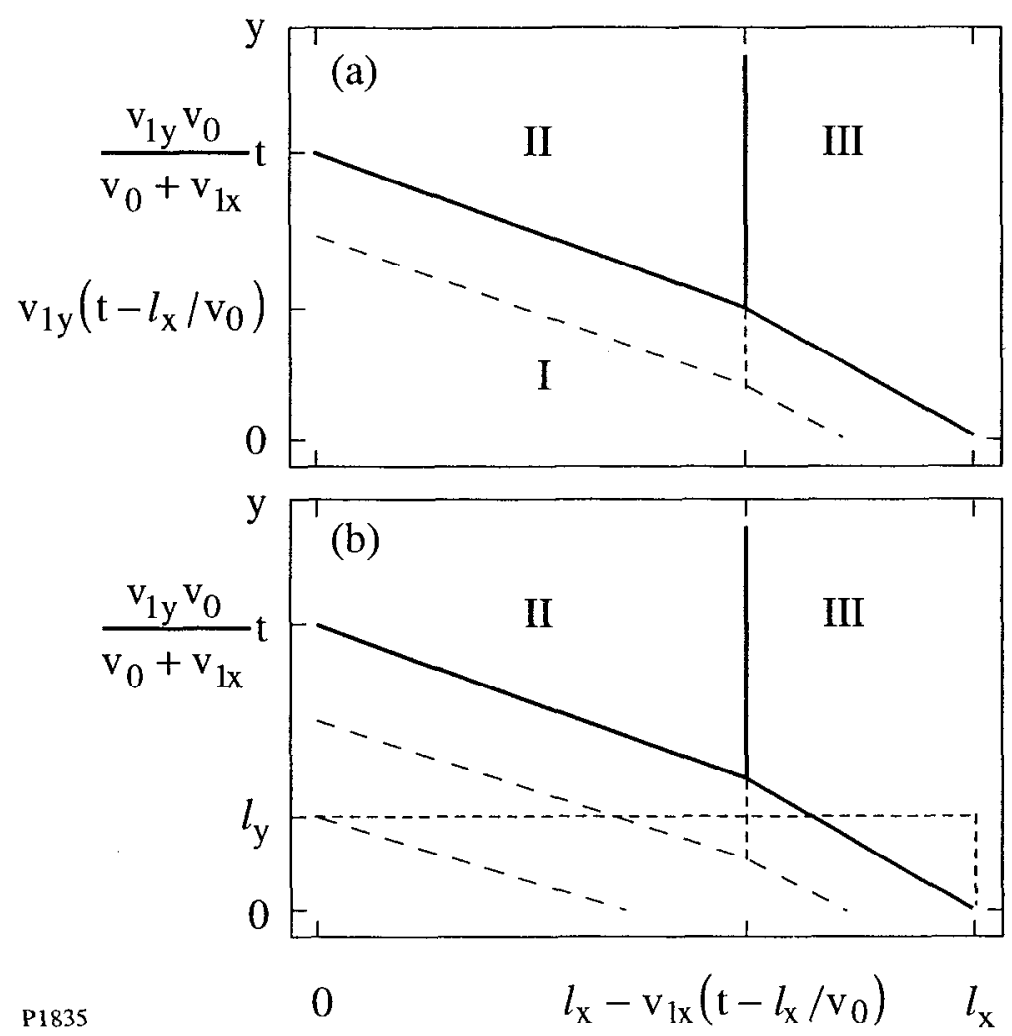

Fig. 4 The three distinct backward scattering regions of growth for each $n$ in Eqs. (13) are divided by the dashed lines. The three distinct general regions of backward scattering growth are divided by the solid lines (which are collinear with the $n=0$ dashed lines). The finite size of the channel is explicitly included in (b). 

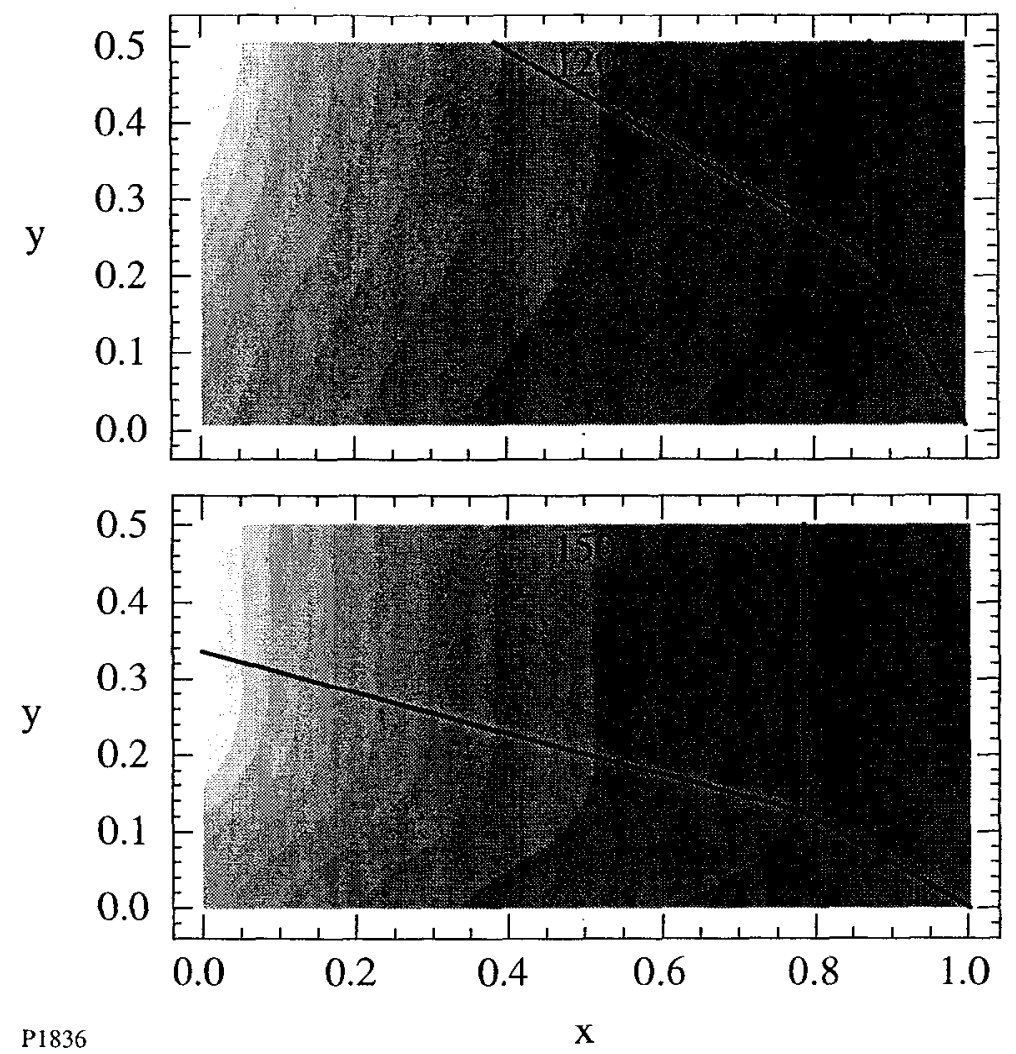

Fig. 5 Linearly shaded contour plots of the Langmuir amplitude [Eqs. (12) and (13)] are shown at time $t=1.25$ for two backward scattering angles. The solid lines distinguish the three general regions of growth. Each plot is normalized so that the largest amplitude is white and smallest amplitude is dark gray. 


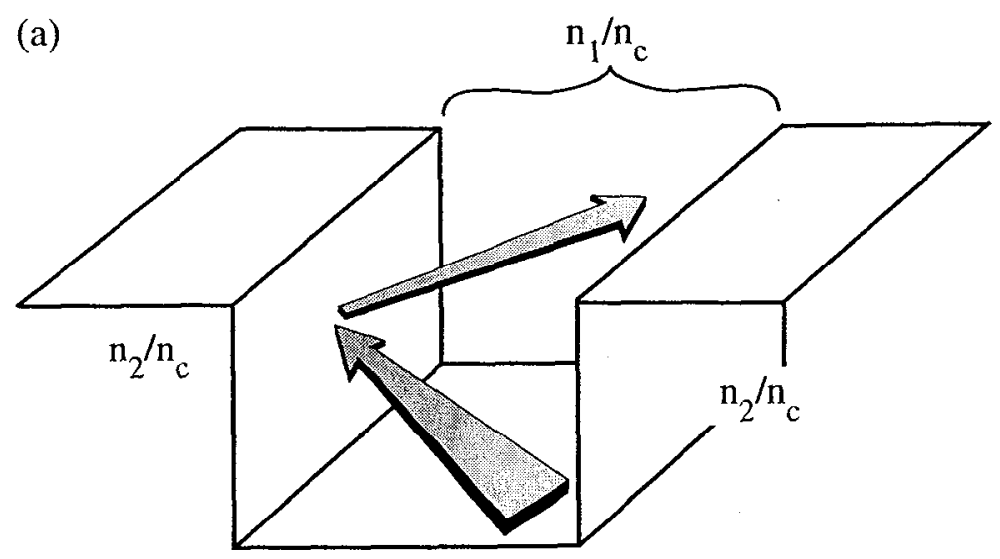

(b)

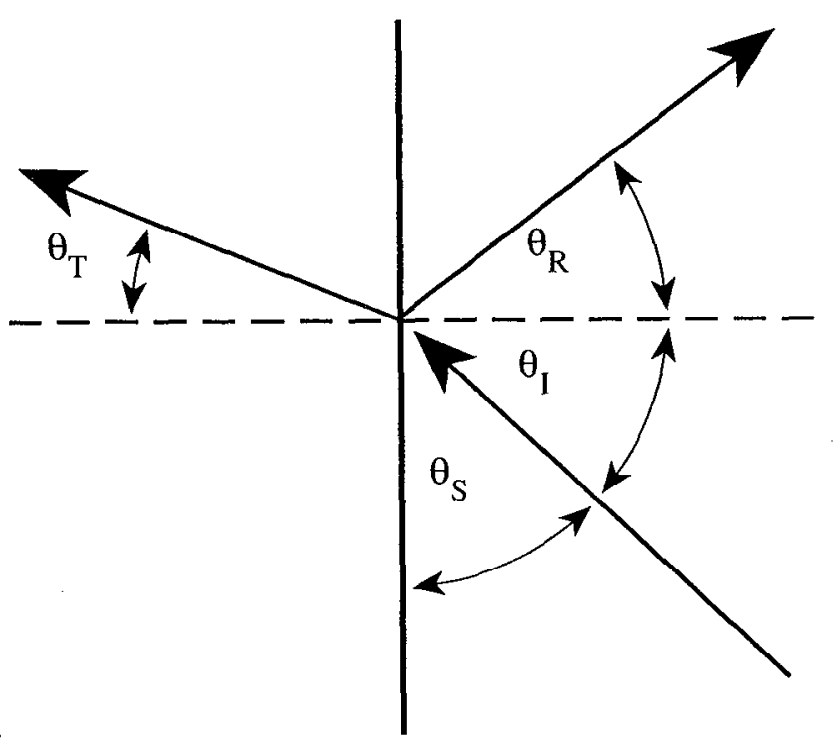

Fig. 6 (a) A basic model for the plasma channel density profile. (b) The scattering geometry at the plasma channel density gradient. 


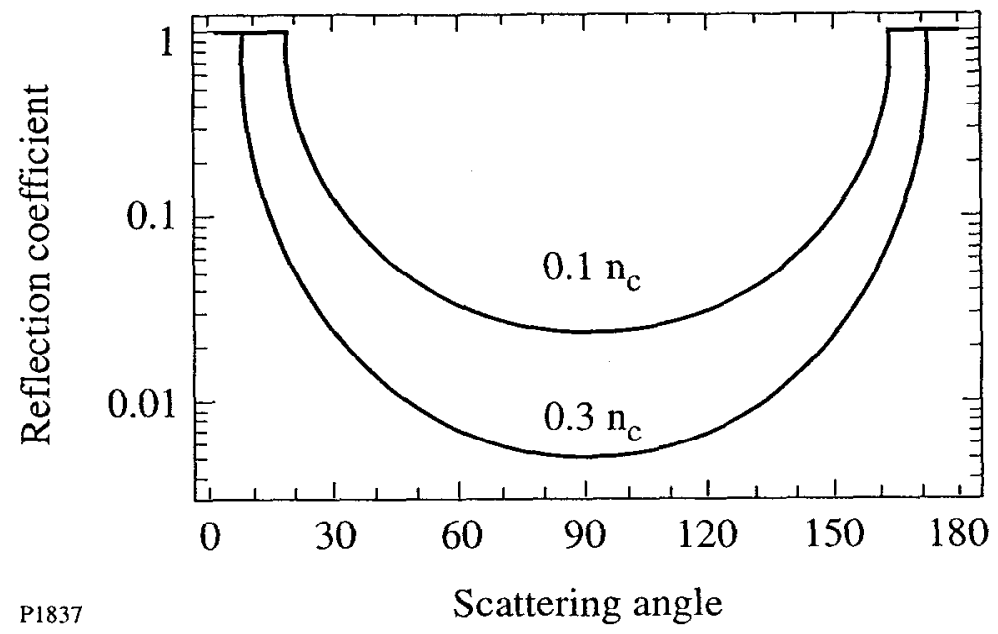

Fig. 7 The reflectivity coefficient [Eq. (20)] is plotted as a function of the Stokes scattering angle for a channel density of $0.01 n_{c}$ and surrounding plasma density of $0.1 n_{c}$ and $0.3 n_{c}$. 


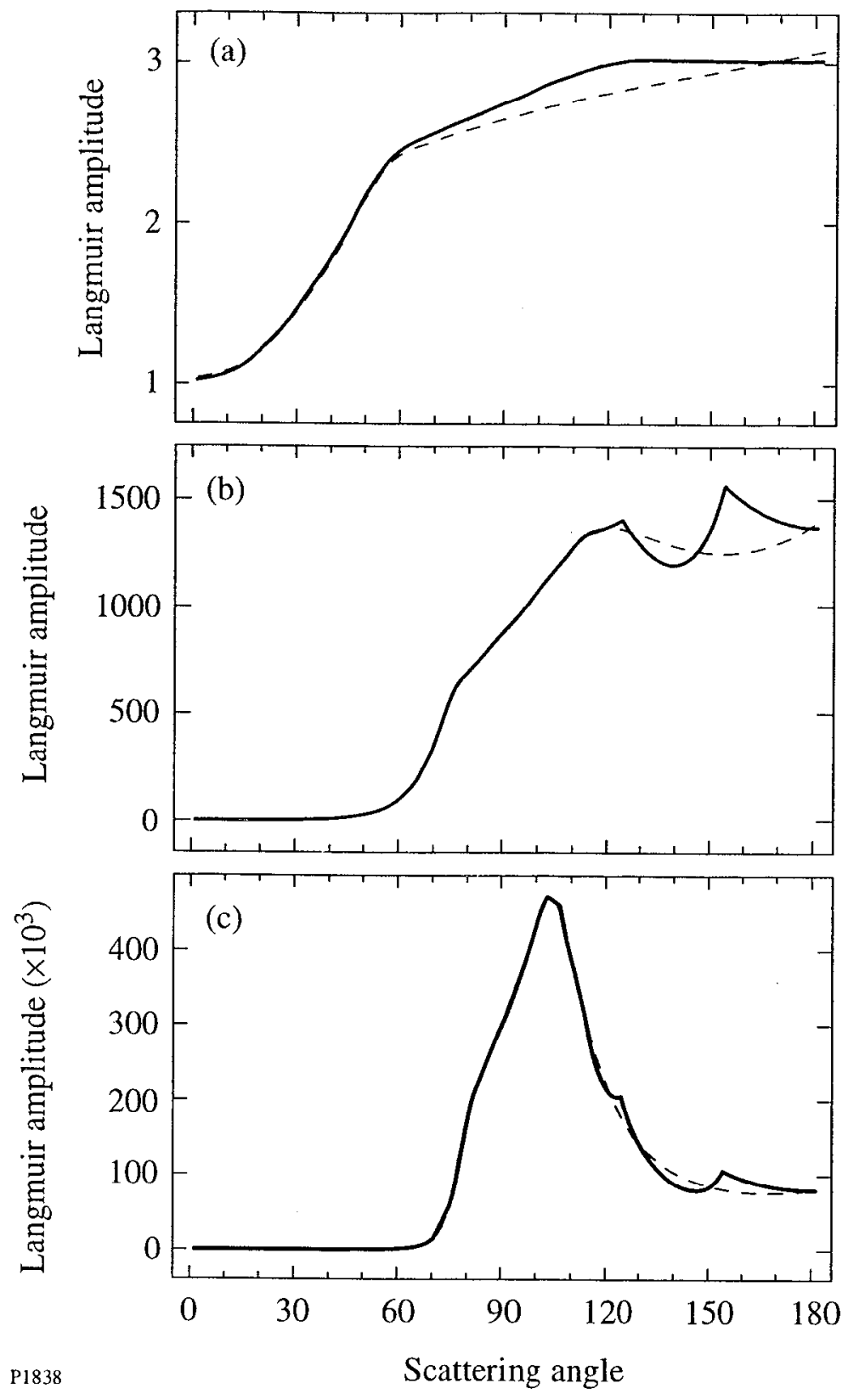

Fig. 8 The exact solution (solid lines) from Eqs. (12) and (13) is compared with the time asymptotic solution (dashed lines) from the one-dimensional damped theory of Ref. 12 for (a) $t=1$, (b) $t=3$, and (c) $t=5$. 'The plasma parameters are the same as in Figs. 3 and 5 . 

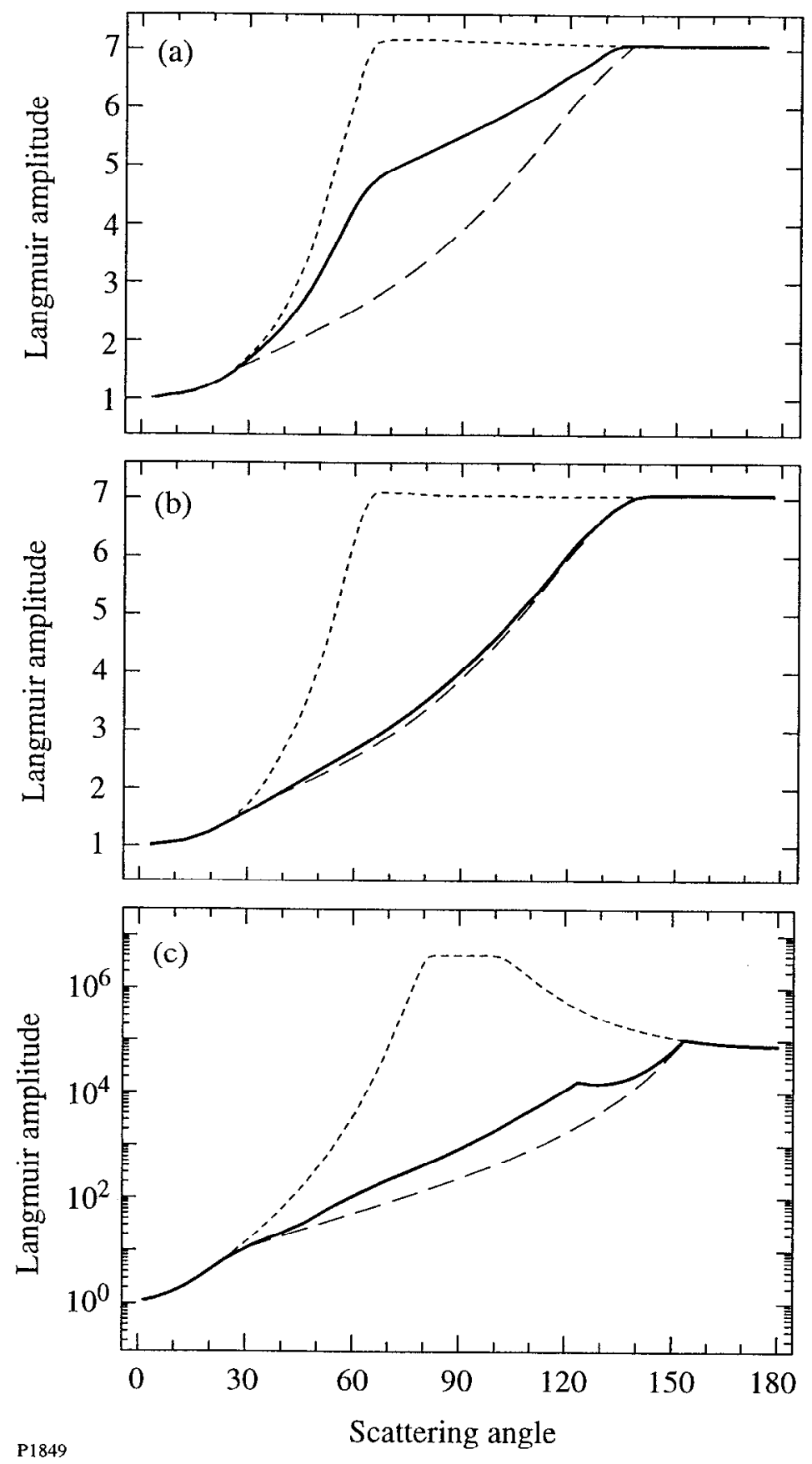

Fig. 9 Comparison of the one dimensional nonreflective theory of Ref. 10 (lines with small dashes), the two-dimensional nonreflective theory of Ref. 12 (lines with large dashes), and the two-dimensional reflective theory developed herein (solid lines). The temporal length of the interaction and reflectivity coefficients are given by: (a) $t=1$, $\mathrm{r}=0.5$, (b) $t=1, r$ given by Eq. (20) with $n_{1}=0.01 n_{c}$ and $n_{2}=$ 0.1 , and (c) $t=5$ with the same reflectivity coefficient as (b). 